\title{
Adjuvant abemaciclib-induced pneumonitis: A case report and review of the literature
}

\author{
ABDULRAHMAN AL-GHABBAN ${ }^{1}$, AISHA AL-SHIBANY ${ }^{1}$, KHADIJA AL-SHANGITI ${ }^{1}$, \\ MOHAMED ASEAFAN $^{1}$, KOUSAY LATIF ${ }^{2}$ and TAHER AL-TWEIGERI ${ }^{1}$ \\ Departments of ${ }^{1}$ Medical Oncology, and ${ }^{2}$ Radiology, \\ King Faisal Specialist Hospital and Research Center, Riyadh 11564, Saudi Arabia
}

Received January 6, 2021; Accepted April 6, 2021

DOI: $10.3892 /$ wasj.2021.99

\begin{abstract}
Cyclin-dependent kinase 4/6 (CDK 4/6) inhibitors have been a major addition to the treatment of hormone receptor-positive, human epidermal growth factor receptor 2 (HER2)-negative breast cancer in the metastatic setting and currently in the adjuvant setting with promising results. However, rare yet potentially fatal side-effects such as interstitial lung disease (ILD)/pneumonitis has been linked to the use of these drugs. The present study describes the case of a female patient with locally advance hormone receptor-positive, HER2-negative breast cancer who developed abemaciclib-induced pneumonitis in the adjuvant setting (monarchE clinical trial). The patient was diagnosed based on presenting with symptoms of cough and shortness of breath, and by a computerized tomography scan after ruling out other
\end{abstract}

Correspondence to: Dr Abdulrahman Al-Ghabban, Department of Medical Oncology, King Faisal Specialist Hospital and Research Center, Makkah Al Mukarramah Branch Road, Al Mathar Ash Shamali, Riyadh 11564, Saudi Arabia

E-mail: aalghabban18@kfshrc.edu.sa

Abbreviations: HR, hormone receptor; HER2, human epidermal growth factor receptor 2; CDK4/6, cyclin-dependent kinase 4/6; CDKs, cyclin-dependent kinases; ILD, interstitial lung disease; $\mathrm{HR}^{+}$, hormonal receptor-positive; HER2, human epidermal growth factor receptor 2-negative; FDA, Food and Drug Administration; SFDA, Saudi Food and Drug Administration; CYP, cytochrome P45; US, ultrasound; CT, computerized tomography; ER, estrogen receptor; $\mathrm{PR}$, progesterone receptor; FISH, fluorescent in situ hybridization; TNM, tumor $(\mathrm{T})$, node $(\mathrm{N})$, and metastasis (M); AJCC, American Joint Committee on Cancer; NYHA, New York Heart Association; CRP, C-reactive protein; PCR, polymerase chain reaction; AFB, acid-fast bacillus; TB, tuberculosis; NCT, National Clinical Trial; PFT, pulmonary function test; DLCO, diffusion capacity of the lungs for carbon monoxide; FVC, forced vital capacity; FEV1, forced expiratory volume in one second; TLC, total lung capacity; ECG, electrocardiogram; ECHO, echocardiogram; IRB, Institutional Review Board

Key words: abemaciclib, cyclin-dependent kinase 4/6, pneumonitis, breast cancer causes. The patient exhibited the resolution of her symptoms completely following the discontinuation of abemaciclib treatment.

\section{Introduction}

Breast cancer is the most frequently diagnosed type of cancer and the leading cause of cancer-related mortality among females globally (1). In general, three major biological subtypes of breast cancer have been well-defined, namely hormonal receptor (HR)-positive $\left(\mathrm{HR}^{+}\right)$, human epidermal growth factor receptor 2 (HER2)-negative (HER2-; luminal A and B type), HER2-postive and triple-negative disease. $\mathrm{HR}^{+} / \mathrm{HER} 2^{-}$breast cancer cases represent between $65-75 \%$ of all breast tumors, and generally respond well to endocrine therapy in both the adjuvant and metastatic setting (2). The activation of cyclin-dependent kinases (CDKs) enables cell cycle progression, representing the hallmark of cancer pathological development. The most important drivers of cell cycle proliferation are known to be CDK4/6 (3). Recently, the US Food and Drug Administration, the European Medicine Agency and the Saudi Food and Drug Administration (SFDA), approved three highly selective inhibitors of CDK4/6 for ( $\left.\mathrm{HR}^{+} / \mathrm{HER} 2^{-}\right)$ advanced metastatic breast cancer, namely (palbociclib, ribociclib and abemaciclib) (4-12). The development and approval of CDKs represent the most relevant advancement in the therapeutic armamentarium and a major milestone in cancer managements over the past decades (13). The addition of these drugs to endocrine therapy has led to a profound improvement of progression-free and overall survival in both first- and second-line indications (14-26).

In general, CDK4/6 inhibitors are well-tolerated and the associated side-effects are easily manageable by early detection, the education of patients, dose reduction and supportive care measures. The most common adverse reactions and hematological toxicities, such as neutropenia are most commonly observed with the use of palbociclib and ribociclib, as opposed to the use of abemaciclib. Ribociclib may cause hepatic toxicity and the prolongation of the QT interval more frequently than other drugs. Abemaciclib is associated with a high prevalence of diarrhea and fatigue in comparison to palbociclib and ribociclib. Hepatotoxicity has also been reported with the use of this molecule, and venous thromboembolic events have 
been reported with the use of abemaciclib compared with the placebo in both the MONARCH2 and MONARCH3 trials (5 vs. $0.9 \%$ ). In addition, increased serum creatinine levels have been reported as a result of the inhibition of several tubular secretion transporters. All three CDKs inhibitors are subjected to interactions with other drugs that are metabolized by or affect the cytochrome P450 (CYP) pathways. For all three agents, the FDA has issued a warning of rare, yet severe cases of fatal lung injury (pneumonitis). Recently, the Japanese Ministry of Health has released a similar warning, as patients developed a pulmonary toxicity, likely secondary to abemaciclib exposure (27-29).

The present study, to the best of our knowledge, is the first to describe the case of a 55-year-old postmenopausal female with locally advanced breast cancer (HR+/HER2- and a Ki67 labeling index of 20\%) who enrolled in the adjuvant high-risk monarchE clinical trial [National Clinical Trial (NCT) 03155997], recently published (30), who developed lung toxicity following treatment with abemaciclib.

\section{Case report}

The present study presents the case of a 55-year-old postmenopausal female with a negative family history of breast or ovarian cancer, who was otherwise healthy, with a right breast tumor mass lasting for $>6$ month in November, 2017. The patient presented at the Combined Breast Oncology Clinic at King Faisal Specialist Hospital and Research Center, Riyadh, Saudi Arabia with a well-defined mass at the 9-10 o'clock position, measuring $\sim 2 \times 2 \mathrm{~cm}$, with multiple axillary lymphadenopathies freely mobile. The patient was subjected to complete staging workup consisting of a bilateral mammogram, an ultrasonography (US) breast scan, a computerized tomography (CT) scan of the chest abdomen and pelvis, and a bone scan as per institutional guidelines. The mammogram and ultrasound revealed 2 highly suspicious masses at the 9-10 o'clock position associated with satellite nodules, one measuring $\sim 2.2 \times 2.3 \mathrm{~cm}$ and the other $1.2 \times 1.2 \mathrm{~cm}$. The overall tumor extension was $\sim 5 \mathrm{~cm}$, with multiple axillary lymph nodes observed, with the largest cortical thickness of $\sim 0.5 \mathrm{~cm}$. A CT scan of the chest, abdomen and pelvis revealed only a right breast mass and multiple axillary lymph nodes (largest of $10 \mathrm{~mm}$ ), no distant metastasis; the bone scan was negative. The biopsy revealed invasive ductal carcinoma of grade 3, estrogen receptor (ER)-positive staining of $95 \%$ and progesterone receptor (PR)-positive staining of $80 \%$; HER 2 was equivocal (+2), as shown by immunohistochemical staining and confirmed negative by fluorescent in situ hybridization (FISH) with ratio of 1.1; the Ki67 labeling index was reported as $70 \%$ (luminal B subtype). The axillary lymph node on the same side was biopsied and reported as positive for carcinoma.

On January 14, 2018, the patient underwent right modified radical mastectomy and the final pathological diagnosis was as follows: Invasive ductal carcinoma, grade 3 , with a multifocal tumor size of 2.6 and $1.5 \mathrm{~cm}, 7$ out 12 lymph nodes positive, with extra-nodal extension, prominent lymphatic and vascular invasion, TNM classification (T2p N2a pM0) and AJCC prognostic Stage Group (IIB). The patient completed four cycles of adjuvant epirubicin and cyclophosphamide $\left(90 / 600 \mathrm{mg} / \mathrm{m}^{2}\right)$ therapy, last administered on the April 18, 2018 with a subsequent 12 weeks of paclitaxel (80 mg/m² day 1 and 8 every 21 days) treatment completed on September 20, 2018. In addition, adjuvant radiation therapy to the right chest wall, the axilla and supraclavicular region, was completed on November 12, 2018. The patient was then selected to be enrolled in the adjuvant high-risk monarchE clinical trial (NCT 03155997) and randomized to receive adjuvant treatment with abemaciclib with exemestane, which commenced on January 30, 2019. The patient was followed-up as per protocol with no major toxicities observed. In June, 2019 the dose of abemaciclib was reduced by one level [100 mg per os (PO) twice daily] due to grade II renal toxicity.

At one year following the initiation of abemaciclib treatment (January, 2020) the patient presented with a dry cough with no other specific symptoms. All the patient workup at that time did not reveal any marked observations, including a chest X-ray; the patient was thus kept on observation. After a period of three months (on April 29, 2020), the patient presented to the Emergency Department of King Faisal Specialist Hospital and Research Center, Riyadh, Saudi Arabia with the same symptom of a dry cough associated with shortness of breath New York Heart Association (NYHA) class II (31) for the past one month. She reported no history of fever, chills or rigors or any contact with patients who were ill or with patients who were COVID-19-positive. She reported no significant travel history and no lower limb edema. Upon examination, she was hemodynamically stable. She did not appear to be in respiratory distress and she was not tachypneic or tachycardic. A chest and cardiovascular examination did not reveal any marked observations and pulse oximetry was normal. The deferential diagnosis at presentation included acute infection, such as pneumonia, pulmonary embolism, lymphangitic carcinomatosis and abemaciclib-induced ILD/pneumonitis. The laboratory workup revealed grade I leukopenia and mild anemia without neutropenia or thrombocytopenia and a stable creatinine clearance $(45-50 \mu \mathrm{mol} / \mathrm{l})$. The chest $\mathrm{x}-$ ray revealed ground-glass opacity in the right upper lobe. C-reactive protein (CRP) and procalcitonin levels were very low. The septic screen was negative for blood cultures. Polymerase chain reaction (PCR) analysis for COVID-19 and common respiratory viruses (rapid influenza $A$ and $B, H 1 N 1$ ), sputum acid-fast bacillus (AFB) cultures and QuantiFERON-TB for tuberculosis (TB) essentially ruled out infectious causes. A CT scan of the chest revealed focal peripheral patchy shadowing of interstitial and air space lung changes noted in the upper and lower lobes of the right lung, in the outer peripheral are in the former and the inner peripheral are in the latter. These lung changes were associated with traction bronchiectasis and a 'tree in bud' appearance, with no evidence of pulmonary embolism, regional recurrence or distant metastasis (Fig. 1). Pulmonary function tests (PFTs) were with good effort and cooperation; [forced vital capacity (FVC), 77\%; forced expiratory volume in the first second (FEV1), 90\%; FEV1/FVC, 116\%; and total lung capacity (TLC), 99\%], revealing no evidence of obstruction or a restrictive pattern. Unfortunately, she was not able to undergo diffusion capacity of the lungs for carbon monoxide (DLCO) according to the ATS standards (32). An electrocardiogram (ECG) and echocardiogram (ECHO) ruled out any cardiac-related causes. The pulmonary team was consulted and they agreed with 
A

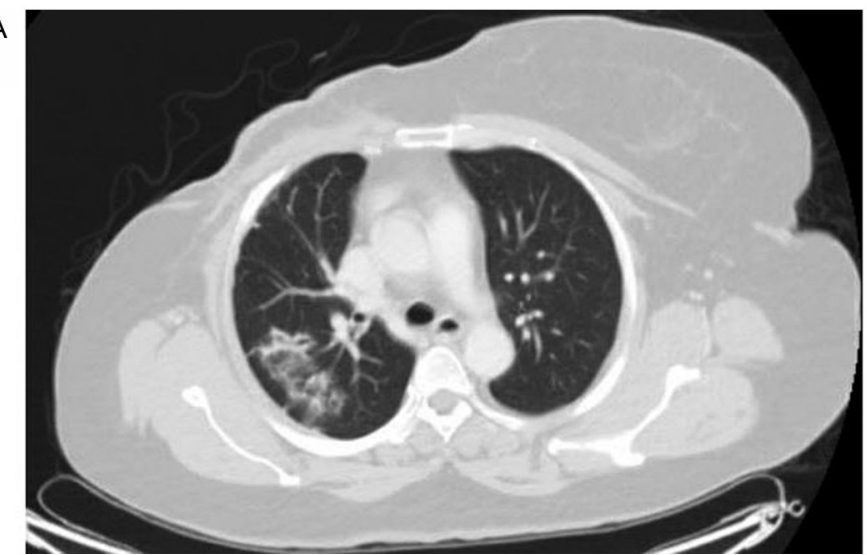

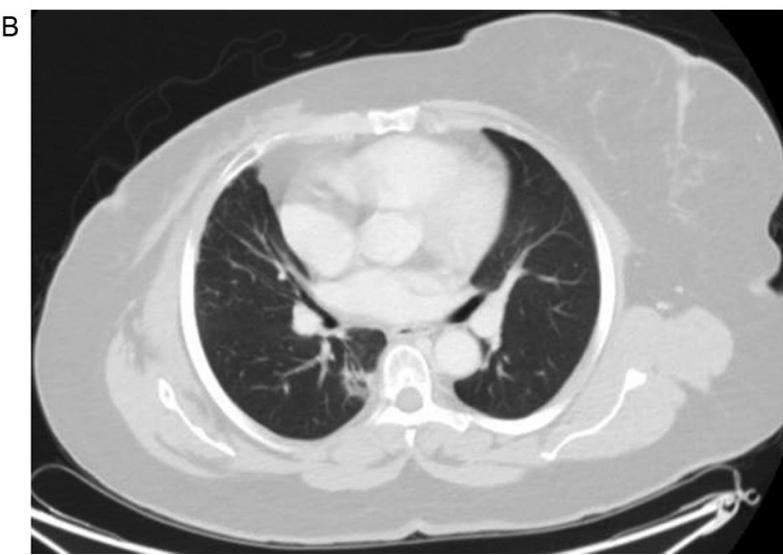

Figure 1. Focal peripheral patchy shadowing of interstitial and air space lung changes noted in the (A) upper and (B) lower lobes of the right lung associated with traction bronchiectasis and a 'tree in bud' appearance.

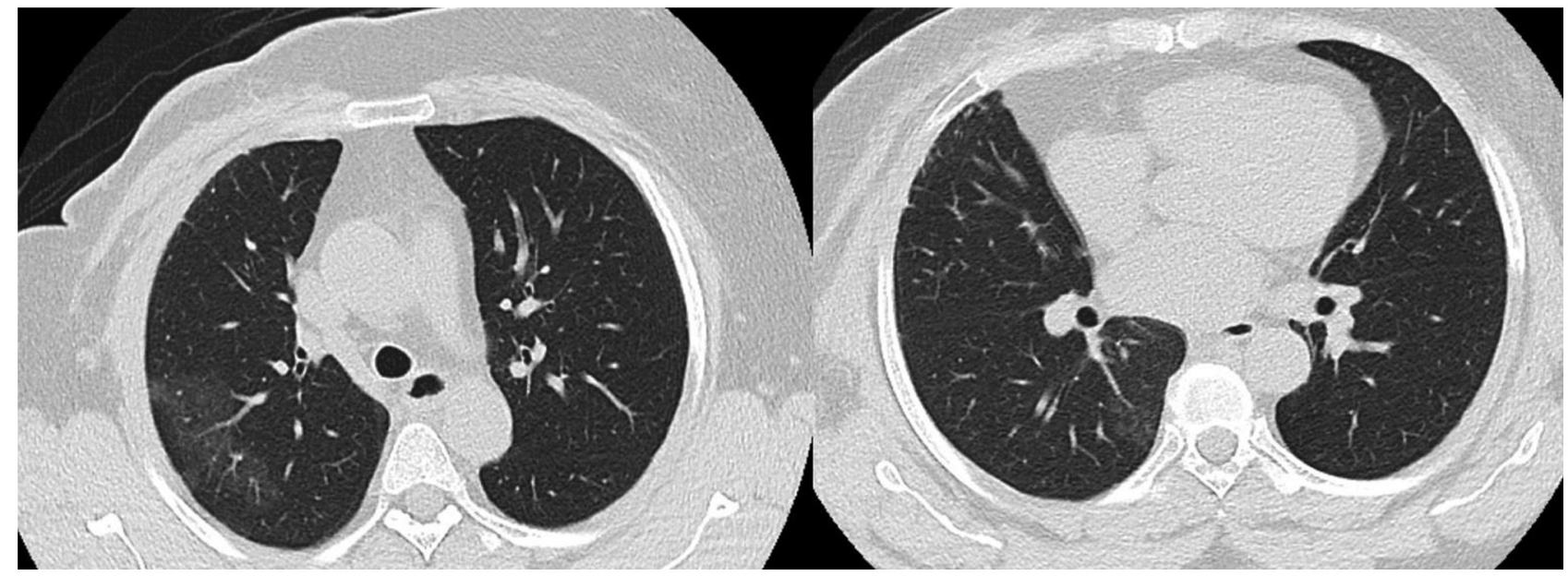

Figure 2. Interval resolution of the focal peribronchovascular ground-glass opacities and consolidations.

the obtained working diagnosis. Abemaciclib treatment was discontinued. The hospital Institutional Review Board (IRB) of King Faisal Specialist Hospital and Research Center and International study group were informed as per the protocol and the patient was kept for observation. Given that the patient had a normal pulmonary function and there were no oxygen requirements, there was no strong indication to begin steroid therapy, and the patient was kept for observation. A follow-up chest $\mathrm{x}$-ray after one week revealed the interval resolution of the noted infiltrates on the admission x-ray that supported the working diagnosis of abemaciclib-induced pneumonitis. The patient was discharged on exemestane. Upon the follow-up after four weeks, the patient had no more symptoms and the CT chest scan at six weeks revealed the interval resolution of the focal peribronchovascular ground-glass opacities and consolidations (Fig. 2).

\section{Discussion}

To date drug-induced, lung injury remains a diagnosis of exclusion. Pulmonary toxicity with anticancer therapy ranging from asymptomatic radiological changes to respiratory failure is considered relatively common with an estimated incidence of $10-20 \%$ prior to the era of CDK4/6 inhibitors (33). The FDA has issued a warning that the use of CDK4/6 inhibitors may cause rare, yet severe inflammation of the lungs. There is far less data available linking CDK4/6 inhibitors with pulmonary toxicity; however, isolated cases from clinical trials plus emerging case reports in the post-marketing arena have raised concern for a potential class effect (29). Initially, there was no report of pneumonitis related to palbociclib in the PALOMA trials (34). To the best of our knowledge, eight cases of palbociclib-induced ILD/pneumonitis have been reported since its approval for advanced $\mathrm{HR}^{+} / \mathrm{HER} 2^{-}$breast cancer (35-42). In the MONALEESA-2 trial of ribociclib, two participants died of acute respiratory failure. In the second interim analysis of the MONALEESA-3 trial there were 6 cases (1.2\%) of ILD [one case $(0.2 \%)$ of grade 3 or 4 ] in the ribociclib arm and 2 cases (none, grade 3 or 4 ) in the placebo arm (22). The authors have recently reported a case of ILD in 46-year-old female with advanced $\mathrm{HR}^{+} / \mathrm{HER} 2^{-}$breast cancer who developed severe acute respiratory distress after four weeks from the commencement of ribociclib therapy (43). Wider knowledge of the risk of pneumonitis related to abemaciclib occurred when the Japanese Health Ministry announced that 14 patients had pulmonary side-effects and three patients died 
following treatment with abemaciclib, after 2,000 patients had been treated with abemaciclib (28). Eli Lilly, the manufacturer of abemaciclib, initially added a warning regarding the risk of ILD, only in their Japanese market, citing a general greater frequency of pneumonitis in the East Asian population (44). However, the FDA later announced, that there were reports of serious cases of pneumonitis and ILD, including fatalities, in patients treated with palbociclib, ribociclib, and abemaciclib, in ongoing clinical trials and post marketing safety databases (29).

Severe, life-threatening, or fatal interstitial lung disease and/or pneumonitis can occur with abemaciclib. Across MONARCH 1, 2 and 3, 3.3\% of abemaciclib-treated patients had ILD of any grade, $0.6 \%$ had grade 3 or 4 , and $0.4 \%$ died (45). Findings from the adjuvant monarchE reported ILD in $2.7 \%$ of patients $(0.3 \%$ grade 3$)$ and given the potential severity of fatal ILD and/or pneumonitis, patients should be made of aware of symptoms and reported immediately to their physicians (30). A recent comprehensive review on the safety analysis of MONARCHE 2 and 3, ILD/pneumonitis was reported as ( 2 and 5.2\%) for MONARCHE 2 and 3, respectively with $<1 \%$ of cases grade $\geq 3$ (46). According to The National Cancer Institute Common Terminology Criteria for Adverse Events (NCI-CTCAE), the patient presented herein fits grade 2 toxicity, which is defined as pneumonitis involving the presence of new or worsening symptoms, including shortness of breath, cough, chest pain, fever and increased oxygen requirements (47). Additionally, the patient developed pneumonitis almost a year following the initiation of adjuvant abemaciclib therapy; the diagnosis of drug-induced lung toxicity was uncertain at the initial presentation, which led to the delay in diagnosis for almost three months. In this case, other differentials were explored (infectious and non-infectious causes) such as, pulmonary embolism; pulmonary edema and heart failure were ruled out by the negative PE scan and microbiology results. Radiation-induced pneumonitis is differential; however, the involvement of both the upper and lower areas away from the field of radiation and the marked improvement that followed the discontinuation of the drug strongly supports abemiclib being the cause. Epirubicin, paclitaxel and cyclophosphamide have been associated with interstitial pneumonia and fibrosis (cyclophosphamide); reportedly, this tends to occur while on therapy $(48,49)$.

The patient presented herein developed interstitial pneumonia almost two years following the completion of the adjuvant chemotherapy, which renders it less likely as the cause. CDK4/6 inhibitors are highly likely to be the causative agent of pulmonary toxicity in several patients who have received the therapy; these agents are new and their side-effects remain poorly understood. As with the treatment of drug-induced pneumonitis, the common recommendation is the discontinuation of the causative agent and to initiate steroids (33). The patient presented herein did not require any oxygen or respiratory support and her symptoms had markedly improved after one week from terminating the medication and the completion of the workup. Therefore, it was determined that the observation of the patient continue with a low threshold to begin steroid treatment upon any change in the respiratory status. It is important for physicians to know that interstitial pneumonitis may be fatal and close observations should be carried out for those patients. CDK4/6 inhibitors may be recognized as the potential cause for drug-induced pneumonitis.

\section{Acknowledgements}

Not applicable.

\section{Funding}

No funding was received.

\section{Availability of data and materials}

Data sharing is not applicable to this article, as no datasets were generated or analyzed during the current study.

\section{Authors' contributions}

AAG contributed to the reviewing of the literature, case description, discussion and writing the initial draft and submission of the final case report. AAS and KAS contributed to the reviewing of the literature and writing of the Introduction. MA contributed to the reviewing of the literature and writing of the Discussion. KL contributed to the case description and in the reviewing of the radiological images. TAT contributed with his guidance to the reviewing and supervising the writing of the case report and final revision. AAG and TAT confirm the authenticity of all raw data. All authors were involved in the design of the study and have read and approved the final manuscript.

\section{Ethics approval and consent to participate}

Not applicable.

\section{Patient consent for publication}

The patient has provided a consent for her data to be published.

\section{Competing interests}

TAT has received speaking honoraria from Roche, Novartis and Lilly, and has served on an advisory committee for Roche, Lilly and Novartis.

\section{References}

1. Bray F, Ferlay J, Soerjomataram I, Siegel RL, Torre LA and Jemal A: Global cancer statistics 2018: GLOBOCAN estimates of incidence and mortality worldwide for 36 cancers in 185 countries. CA Cancer J Clin 68: 394-424, 2018.

2. National Cancer Institute: SEER Cancer Statistics Review, 1975-2016. https://seer.cancer.gov/archive/csr/1975_2016/. Accessed: June 30, 2020.

3. de Melo Gagliato D, C Buzaid A, Perez-Garcia JM, Llombart A and Cortes J: CDK4/6 Inhibitors in hormone receptor-positive metastatic breast cancer: Current practice and knowledge. Cancers (Basel) 12: 2480, 2020.

4. U.S. Food and Drug: FDA approves abemaciclib as initial therapy for HR-positive, HER2-negative metastatic breast cancer. https:// www.fda.gov/drugs/resources-information-approved-drugs/fda-a pproves-abemaciclib-initial-therapy-hr-positive-her2-negative-m etastatic-breast-cancer. Accessed March 27, 2021. 
5. U.S. Food and Drug: FDA apporves palbociclib as a treatment of hormone receptor (HR) positive, human epidermal growth factor receptor 2 (HER2) negative advanced or metastatic breast cancer in combination with an aromatase inhibitor as initial endocrine based therapy in postmenopausal women; https://www fda.gov/drugs/resources-information-approved-drugs/palbociclib-ibrance. Accessed March 27, 2021.

6. U.S. Food and Drug: FDA approved ribociclib, a cyclin-dependent kinase 4/6 inhibitor, in combination with an aromatase inhibitor as initial endocrine-based therapy for the treatment of postmenopausal women with hormone receptor (HR)-positive, human epidermal growth factor receptor 2 (HER2)-negative advanced or metastatic breast cancer; https://www.fda.gov/drugs/resourcesinformation-approved-drugs/ribociclib-kisqali. Accessed March 27, 2021.

7. European Medicine Agency (EMA): EMA approval of palbociclib; https://www.ema.europa.eu/en/medicines/human/EPAR/ibrance. Accessed March 27, 2021.

8. European Medicine Agency (EMA): EMA approval of ribociclib; https://www.ema.europa.eu/en/medicines/human/EPAR/kisqali. Accessed March 27, 2021.

9. European Medicine Agency (EMA): EMA approval of abemaciclib; https://www.ema.europa.eu/en/medicines/human/EPAR/verzenios. Accessed March 27, 2021.

10. Saudi FDA: Approval and registration of palbociclib; https://sdi.sfda.gov.sa/Home/Result?drugId=7121. Accessed March 28, 2021.

11. Saudi FDA: Approval and registration of ribociclib; https://sdi.sfda.gov.sa/Home/Result?drugId=7956. Accessed March 28, 2021.

12. Saudi FDA: Approval and registration of abemaciclib; https://sdi.sfda.gov.sa/Home/Result?drugId=8132. Accessed March 28, 2021.

13. Piezzo M, Cocco S, Caputo R, Cianniello D, Gioia GD, Lauro VD Fusco G, Martinelli C, Nuzzo F, Pensabene M and Laurentiis M: Targeting cell cycle in breast cancer: CDK4/6 inhibitors. Int J Mol Sci 21: 6479, 2020.

14. Chen X, Xu D, Li X, Zhang J, Xu W, Hou J, Zhang W and Tang J: Latest overview of the cyclin-dependent kinases $4 / 6$ inhibitors in breast cancer: The past, the present and the future. J Cancer 10 6608-6617, 2019

15. Gao JJ, Cheng J, Bloomquist E, Sanchez J, Wedam SB, Singh H, Amiri-Kordestani L, Ibrahim A, Sridhara R, Goldberg KB, et al: CDK4/6 inhibitor treatment for patients with hormone receptor-positive, HER2-negative, advanced or metastatic breast cancer: A US food and drug administration pooled analysis. Lancet Oncol 21: 250-260, 2020.

16. Hortobagyi GN, Stemmer SM, Burris HA, Yap YS, Sonke GS, Paluch-Shimon S, Campone M, Petrakova K, Blackwell KL, Winer EP, et al: Updated results from MONALEESA-2, a phase III trial of first-line ribociclib plus letrozole versus placebo plus letrozole in hormone receptor-positive, HER2-negative advanced breast cancer. Ann Oncol 29: 1541-1547, 2018.

17. Tripathy D, Im SA, Colleoni M, Franke F, Bardia A, Harbeck N, Hurvitz SA, Chow L, Sohn J, Lee KS, et al: Ribociclib plus endocrine therapy for premenopausal women with hormone-receptor-positive, advanced breast cancer (MONALEESA-7): A randomised phase 3 trial. Lancet Oncol 19: 904-915, 2018

18. Hurvitz SA, Im SA, Lu YS, Colleoni M, Franke FA, Bardia A, Harbeck N, Chow L, Sohn J, Lee KS, et al: Phase III MONALEESA-7 trial of premenopausal patients with HR+/HER2-advanced breast cancer (ABC) treated with endocrine therapy \pm ribociclib: Overall survival (OS) results. J Clin Oncol 37: LBA1008, 2019.

19. Slamon DJ, Neven P, Chia S, Fasching PA, De Laurentiis M, Im SA, Petrakova K, Bianchi GV, Esteva FJ, Martín M, et al: Phase III randomized study of ribociclib and fulvestrant in hormone receptor-positive, human epidermal growth factor receptor 2-negative advanced breast cancer: MONALEESA-3. J Clin Oncol 36: 2465-2472, 2018

20. Slamon DJ, Neven P, Chia S, Fasching PA, De Laurentiis M, Im SA, Petrakova K, Bianchi GV, Esteva FJ, Martin M, et al: Overall survival (OS) results of the phase III MONALEESA-3 trial of postmenopausal patients (pts) with hormone receptorpositive $(\mathrm{HR}+)$, human epidermal growth factor 2-negative (HER2-) advanced breast cancer (ABC) treated with fulvestrant (FUL) \pm ribociclib (RIB). Ann Oncol 30: V856-V857, 2019 .
21. Slamon DJ, Neven P, Chia SKL, Im SA, Fasching PA, DeLaurentiis M, Petrakova K, Bianchi GV, Esteva FJ, Martin M, et al: Ribociclib (RIB) + fulvestrant (FUL) in postmenopausal women with hormone receptor-positive (HR+), HER2-negative (HER2-) advanced breast cancer (ABC): Results from MONALEESA-3. J Clin Oncol 36: 1000, 2018.

22. Slamon DJ, Neven P, Chia S, Fasching PA, De Laurentiis M, Im SA, Petrakova K, Bianchi GV, Esteva FJ, Martín M, et al: Overall survival with ribociclib plus fulvestrant in advanced breast cancer. N Engl J Med 382: 514-524, 2020.

23. Lu YS, Bardia A, Vázquez RV, Colleoni MA, Wheatley-Price P, Im YH, Babu G, Tripathy D, Lanoue BR, Chandiwana D, et al: Updated overall survival (OS) and quality of life (QoL) in premenopausal patients (pts) with advanced breast cancer (ABC) who received ribociclib (RIB) or placebo (PBO) plus goserelin and a nonsteroidal aromatase inhibitor (NSAI) in the MONALEESA-7 (ML-7) trial. Ann Oncol 30: V106, 2019.

24. Im SA, Lu YS, Bardia A, Harbeck N, Colleoni M, Franke F, Chow L, Sohn J, Lee KS, Campos-Gomez S, et al: Overall Survival with ribociclib plus endocrine therapy in breast cancer. N Engl J Med 381: 307-316, 2019.

25. Sledge GW, Toi M, Neven P, Sohn J, Inoue K, Pivot X, Burdaeva O, Okera M, Masuda N, Kaufman PA, et al: MONARCH 2: Overall survival of abemaciclib plus fulvestrant in patients with $\mathrm{HR}+$, HER2- advanced breast cancer. Ann Oncol 30: V856, 2019.

26. Sledge GW Jr, Toi M, Neven P, Sohn J, Inoue K, Pivot X, Burdaeva O, Okera M, Masuda N, Kaufman PA, et al: The Effect of abemaciclib plus fulvestrant on overall survival in hormone receptor-positive, ERBB2-negative breast cancer that progressed on endocrine therapy-MONARCH 2: A randomized clinical trial. JAMA Oncol 6: 116-124, 2019.

27. Spring LM, Wander SA, Andre F, Moy B, Turner NC and Bardia A: Cyclin-dependent kinase 4 and 6 inhibitors for hormone receptor-positive breast cancer: Past, present, and future. Lancet 395: 817-827, 2020.

28. Breast cancer drug Verzenio could have serious side effects on lungs Js, health ministry warns. The Japan Times. 17 May, 2019.

29. U.S. Food and Drug: FDA warns about rare but severe lung inflammation with Ibrance, Kisqali, and Verzenio for breast cancer. https://www.fda.gov/drugs/drug-safety-and-availability/fda-warn s-about-rare-severe-lung-inflammation-ibrance-kisqali-and-verz enio-breast-cancer. Accessed September 9, 2019

30. Johnston SRD, Harbeck N, Hegg R, Toi M, Martin M, Shao ZM, Zhang QY, Martinez Rodriguez JL, Campone M, Hamilton E, et al: Abemaciclib combined with endocrine therapy for the adjuvant treatment of HR+, HER2-, Node-positive, high-risk, early breast cancer (monarchE). J Clin Oncol 38: 3987-3998, 2020

31. American Heart Association: Classes of Heart Failure; https://www.heart.org/en/health-topics/heart-failure/what-is-heartfailure/classes-of-heart-failure. Accessed March 27, 2021.

32. American Thoracic Society: 2017 ERS/ATS standards for single-breathcarbon monoxide uptake in the lung; https://www. thoracic.org/statements/resources/pft/DLCO.pdf. Accessed March 27, 2021.

33. Omarini C, Thanopoulou E and Johnston SR: Pneumonitis and pulmonary fibrosis associated with breast cancer treatments. Breast Cancer Res Treat 146: 245-258, 2014.

34. Finn RS, Martin M, Rugo HS, Jones S, Im SA, Gelmon K, Harbeck N, Lipatov ON, Walshe JM, Moulder S, et al: Palbociclib and letrozole in advanced breast cancer. N Engl J Med 375: 1925-1936, 2016.

35. Ahsan I, Malik F and Jafri SI: Palbociclib related pnemotoxicity: A rare side effect. In: C43. Drug Induced Lung Disease: Case Reports, ppA5546, 2017.

36. Azhar A, Zaman K and Samad R: A tale of unexplained pneumotoxicity: The dangers of palbociclib. In: B36. Case Reports in Biologic and Chemotherapeutic Agent Induced Lung Disease, ppA3120, 2020.

37. Felip E, Llobera L, Perez-Mañá C, Quintela D, Guasch I, Margelí M, Teruel I, Cirauqui B, Centeno C, Romeo M, et al: New drugs, old toxicities: Pneumonitis related to palbociclib-a case report. Breast Care 15: 548-552, 2020.

38. Gong J, Cho M, Yu KW, Waisman J, Yuan Y and Mortimer J: A single institution experience with palbociclib toxicity requiring dose modifications. Breast Cancer Res Treat 168: 381-387, 2018.

39. Jazieh KA, Budd GT, Dalpiaz N and Abraham J: Can CDK4/6 inhibitors cause fatal lung injury? Expert Rev Anticancer Ther 19: 917-919, 2019. 
40. Levy O, Ptashkin E, Shechtman Y, Kas T, Natif N, Sanset S and Katz D: Fatal palbociclib-related interstitial pneumonitis. Arch Clin Med Case Rep 3: 162-166, 2019.

41. Okura F, Sato Y, Murakami E, Komatsu H, Yamamura Y and Ito Y: A case of interstitial pneumonitis induced by palbociclib. Gan To Kagaku Ryoho 47: 997-999, 2020 (In Japanese).

42. Sarkisian S, Markosian C, Ali Z and Rizvi M: Palbociclibinduced pneumonitis: A case report and review of the literature. Cureus 12: e8929-e8929, 2020.

43. Algwaiz G, Badran AA, Elshenawy MA and Al-Tweigeri T: Ribociclib-induced pneumonitis: A case report. Breast Care: https://doi.org/10.1159/000507647.

44. Liu A: Lilly oncology suffers another blow as Verzenio slapped with safety warning in Japan. Fierce Pharma, 2019. https://www. fiercepharma.com/pharma/lilly-oncology-suffers-another-blow -as-verzenio-slapped-safety-warning-japan. Accessed May 21, 2019 ,

45. Eli Lilly and Company: Abemaciclib (Verzenio R) US prescribing information. http://pi.lilly.com/us/verzenio-uspi.pdf. Accessed September 2019
46. Rugo HS, Huober J, García-Sáenz JA, Masuda N, Sohn JH Andre VAM, Barriga S, Cox J and Goetz M: Management of abemaciclib-associated adverse events in patients with hormone receptor-positive, human epidermal growth factor receptor 2-negative advanced breast cancer: Safety analysis of MONARCH 2 and MONARCH 3. Oncologist 26: e522-e522, 2021.

47. Chuzi S, Tavora F, Cruz M, Costa R, Chae YK, Carneiro BA and Giles FJ: Clinical features, diagnostic challenges, and management strategies in checkpoint inhibitor-related pneumonitis. Cancer Manag Res 9: 207-213, 2017.

48. Kawajiri H, Takashima T, Onoda N, Kashiwagi S, Ishikawa T and Hirakawa K: Interstitial pneumonia associated with neoadjuvant chemotherapy in breast cancer. Mol Clin Oncol 1: 433-436, 2013.

49. Bielopolski D, Evron E, Moreh-Rahav O, Landes M, Stemmer SM and Salamon F: Paclitaxel-induced pneumonitis in patients with breast cancer: Case series and review of the literature. J Chemother 29: 113-117, 2017. 